\section{Embedding Small Specimens for TEM Examination}

\section{Paul Webster \\ House Ear Institute \\ pwebster@hei.org}

When preparing specimens for TEM analysis, it is important that specimens routinely be large enough to manipulate with forceps and cohesive enough to hold together as a single entity. However, anyone working in EM will have some specimens that are too small to see or centrifuged pellets that easily fall apart during processing. These specimens can easily be lost during a routine dehydration and embedding protocol and therefore cannot be sectioned. If the specimen to be processed is too small to handle or will fall apart, then precautions can be taken to ensure that it can be safely embedded for sectioning. Although this article is written with the main focus on biomedical electron microscopy, these handling protocols can be applied to any small specimens that have to be thin sectioned. Embedding can be performed in resin or any other support medium.

\section{Embedding in agarose}

Many small specimens, and even fragile tissues and materials can be embedded in a $2 \%$ solution of low-melting-point agarose. Biological materials are chemically crosslinked in buffered aldehyde and then washed in buffer containing a small amount of glycine to neutralize the aldehyde. The small specimens are then immersed in a warm, and thus liquid, solution of agarose. When cooled, the block can be suitably trimmed and processed using routine dehydration and resin embedding protocols.

This approach has worked well for embedding embryos, cells and cell fragments in resin and for cryosectioning (Bierkamp et al., 1999; Hook \& Svalander 1991; Pedersen et al., 2000). The method does have disadvantages. Even low melting point agarose exposes specimens to elevated temperatures, which may affect antigenicity, or if labile materials are being examined, the specimen may melt. Also many specimens are either too small, or the agarose is too opaque, for easy visualization and trimming. For these instances, other methods can be used.

\section{Increasing the volume of the pellet}

Another method for handling very small specimens is to bulk up the volume of the specimen with something that can be easily recognized in the section and ignored. For biological specimens, a small drop of red blood cells can be added to cells or cell fragments. The mixture is then centrifuged down into a tight pellet for subsequent handling. Very small amounts of the added material have to be used or the specimen can be lost in the extra mass. Alternatively, red cell ghosts, or even small amounts of keratin (from skin flakes) can be used to bulk up the pellet.

An advanced version of this pelleting method uses agarose or sepharose beads to bulk up the pellet. If affinity markers (antibodies or other molecules with specific binding abilities) are attached to the surface of the beads, then cells or cell fragments can be affinity purified and detected on the bead surface. Immobilized structures on the bead surface can subsequently be labeled with other affinity markers using pre-embedding labeling protocols.

Another approach to increasing the pellet volume, which also incorporates a cross-linking step is to embed the specimen in a fibrin clot (Raska et al., 1998). The specimen is mixed with a small amount of fibrin and then reacted with fibrinogen to form a clot around the specimen. Specimens can be centrifuged down into a pellet before clotting or if they are too fragile, they can be left suspended in the clot.

\section{Embedding on a solid substrate}

Another, specialized way of handling small pellets and even single cells, is to attach the specimen onto a glass or plastic coverslip. Although regular coverslips can be used, it is easier to locate small structures if the coverslip has a grid etched into its surface. Coat the grid with something that will stick the specimen down (e.g. poly-L-lycine or alcian blue) and let the specimen settle onto the surface (material scientists may find other substances are more suitable for attaching small fragments onto glass). The specimen should be attached to the same side as the etched grid. Fix, dehydrate and embed the region where the specimen is attached in resin. The polymerized resin block (with, or without the coverslip, as the grid pattern is transferred to the resin) can be examined by light microscopy and specific regions can be identified and sectioned. It is possible to centrifuge specimens down onto the glass surface using a cytospin centrifuge. However, unfixed specimens should not be allowed to dry out. We have successfully used this approach to examine protozoans (Gardiner et al., 1986)
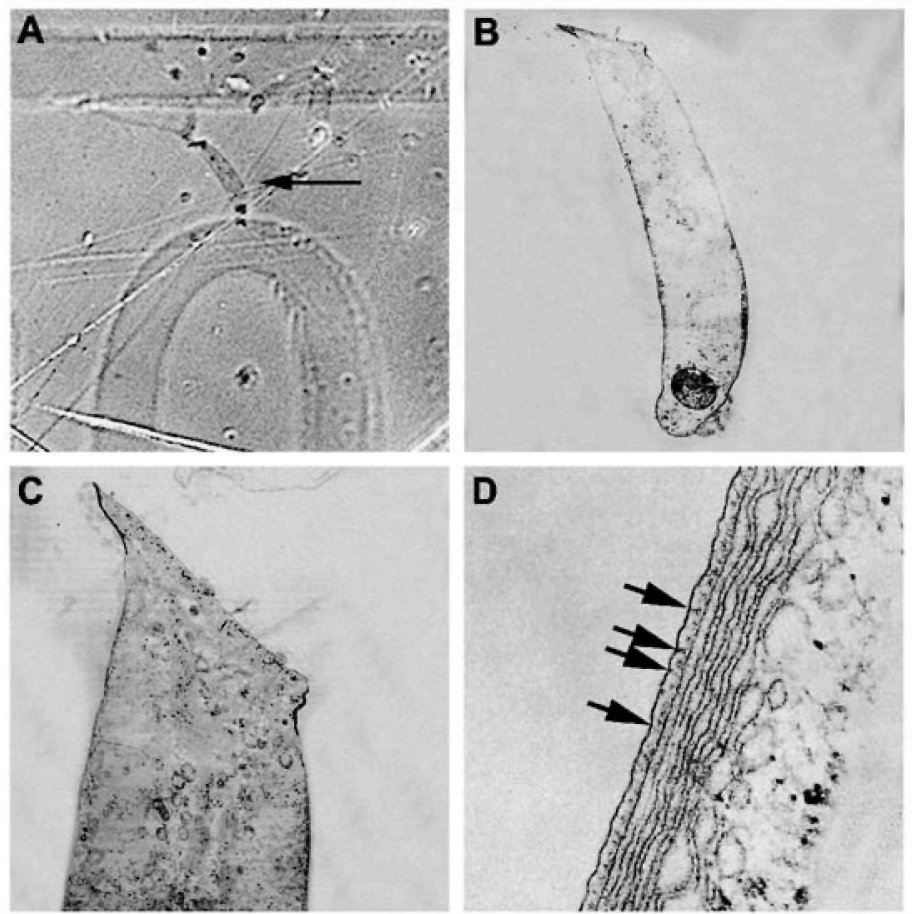

Figure 1. Electron microscopy of a single cell. This is an example of how a single outer hair cell can be embedded in resin, identified by light microscopy and sectioned for examination in the transmission electron microscope. Outer hair cells were isolated from the organ of Corti by physical dissection and enzyme treatment. The separated cells were left to settle on a glass coverslip on which had been etched a locator grid. The glass coverlip was then immersed in $2.5 \%$ buffered ghutaraldehyde, washed, exposed to $1 \%$ osmium tetroxide, dehydrated in ethanol and propylene oxide and finally embedded in epoxy resin. The glass was removed from the polymerized resin block by immersion in liquid nitrogen, the exposed surface cleaned with $70 \%$ ethanol and the block examined by phase contrast light microscopy. Figure 1A. The image observed by phase contrast light microscopy. An individual outer hair cell can be observed in the resin (arrow). Marks on the resin surface and the locator grid mark (in this instance the cell was located in square O5, part of the letter " $O$ ' is visible in the image. Figures $1 B-D$. The outer hair cell located in Figure $1 A$ was identified and the resin around it trimmed away. The block was glued onto a firm support (in this instance an empty resin block was used) and placed into an ultramicrotome. Thin sections approximately $70 \mathrm{~nm}$ thick were produced, collected on metal specimen grids, contrasted and examined in a transmission electron microscope. The arrows in Figure 1D highlight small bridges on the cell periphery that appear to connect the outer cell membrane with inner membranes that are presumed to be rough endoplasmic reticulum. It is clear that single cells can be identified in resin blocks and sectioned for electron microscopy. Subcellular detail is only affected by the mechanical damage that occurs before the cells are chemically cross-linked. 



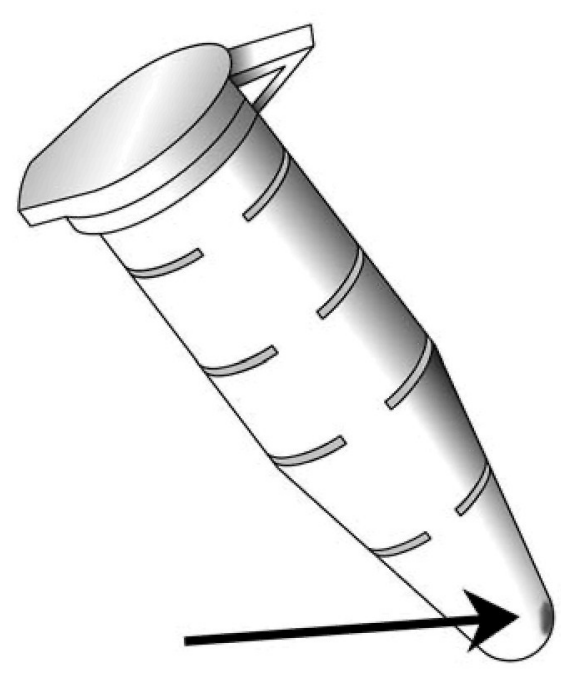

Figure 2. Centrifugation of small specimens. If the hinge of an Eppendorf tube is placed uppermost in $a 5^{\circ}$ fixed angle rotor, the material in the tube will always be centrifuged to the same spot below the hinge. embedding that make it useful for even novice users. A $10 \%$ solution of gelatin will be liquid at $37^{\circ} \mathrm{C}$ so specimens to be embedded are never exposed to high temperatures (as may be the case with agarose embedding). If it is determined that specimens are incorrectly embedded after the gelatin has solidified, then the solution only needs to be re-warmed to $37^{\circ} \mathrm{C}$ for it to become liquid again. Gelatin can easily be removed from embedded specimens, or from sections, if required, by washing with warm aqueous medium.

Unlike agarose, which encases specimens, gelatin tends to stick to biological specimens and gives extra support. This is especially important if the specimen has to be cut into smaller pieces after being encased. Cells and tissues will fall out of agarose when it is handled too much, but gelatin remains attached to, and between, the specimen.

Really small specimens are chemically crosslinked with buffered aldehyde, treated with glycine and placed in a thin polypropylene centrifuge tube (about $0.5 \mathrm{ml}$ total volume). The specimens are gently centrifuged to the bottom of the tube, re-suspended in a small volume of warm $10 \%$ gelatin and centrifuged again to form a tight pellet (Amigorena et al., 1994). It may still be possible to obtain centrifuge tubes with sharp pointy ends on the inside surface. These work best because the pellet fragments are deposited in the very tip of the tube when centrifuged using a horizontal rotor.

After the gelatin had gelled, cut off the tip of the tube, which is easy if the tube is made of polypropylene, and embed using a routine protocol. For cryosectioning, we soak the gelatin block in $2.3 \mathrm{M}$ sucrose and freeze by immersion in liquid nitrogen. For embedding biological specimens in epoxy resin we fix the gelatin in $2 \%$ buffered glutaraldehyde, post fix with osmium tetroxide and dehydrate in ethanol. The gelatin turns dark and the tip remains visible making orientation of the block a simple procedure.

An alternative centrifugation step of small specimens or cell fragments is to centrifuge them down, in
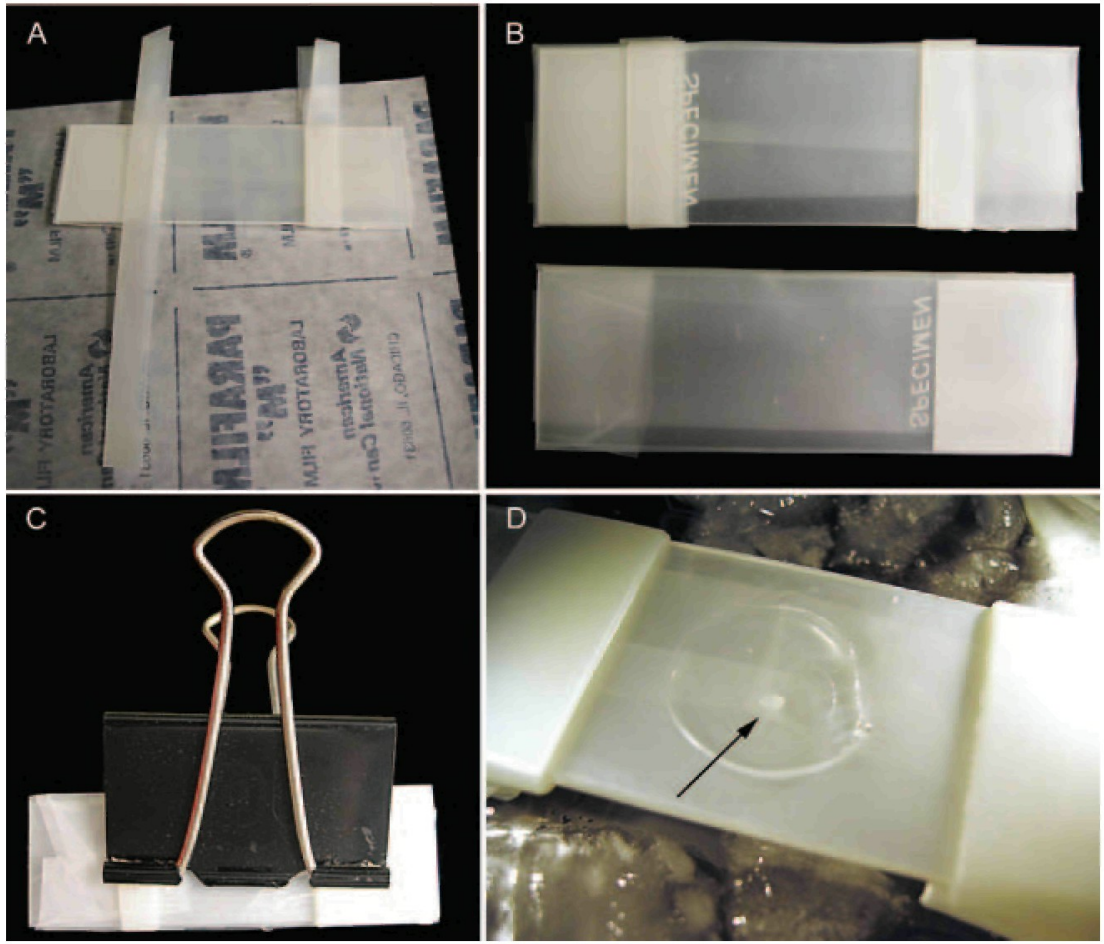

Figure 3. Embedding in gelatin. Small specimens can be placed between two Parafilm $M^{\text {ra }}$ covered glass slides. The slides are completely wrapped in sheets of Parafilm $M^{\circ \times}$. One of the glass slides is then wrapped again with folded strips of Parafilm $M^{\oplus \mathrm{r}}$ to act as spacers (FIGs $3 \mathrm{~A}$ and $B)$. The small specimen is then placed on the slide with spaces, covered with warm $10 \%$ gelatin and covered with the second slide. The two slides are held in place with a clamp (FIG 3C) and left at $4^{\circ} \mathrm{C}$ until the gelatin has set. When the two slides are separated (FIG 3D), the specimen (indicated in FIG $3 D$ with an arrow) will be embedded in a thin film of solidified gelatin. If the gelatin is kept on ice it will remain solid and the specimen can be easily cut out of the gelatin sheet for further processing. (Many thanks to Dr Eija Jokitalo of the EM Unit at the University of Helsinki for introducing me to this method of embedding cells). 
cytes. Nature 369:113-120.

2. Bierkamp, C., H. Schwarz, O. Huber, and R. Kemler. 1999. Desmosomal localization of b-catenin in the skin of plakoglobin null-mutant mice. Development 126, 371-381.

3. Gardiner, P. R., P. Webster, L. Jenni and S. K. Moloo. 1986. Metacyclic Trypanosoma vivax posses a surface coat. Parasitology. 92: 75-82.

4. Hook U. and C.T. Svalander. 1991. Agarose method for the preparation of isolated glomeruli from human renal biopsies. APMIS. 1991 99:844-848.

5. Pedersen K, E.J. Snijder, S. Schleich, N. Roos, G. Griffiths and, J.K. Locker. 2000 Characterization of vaccinia virus intracellular cores: implications for viral uncoating and core structure. J Virol. 74:3525-3536.

6. Raska I, A. Pliss, V. Mandys, M.C. Risueno and Z. Lojda 1998. Processing of free cells for electron microscopy using a fibrin clot. Acta Histochem. 100:309-313.

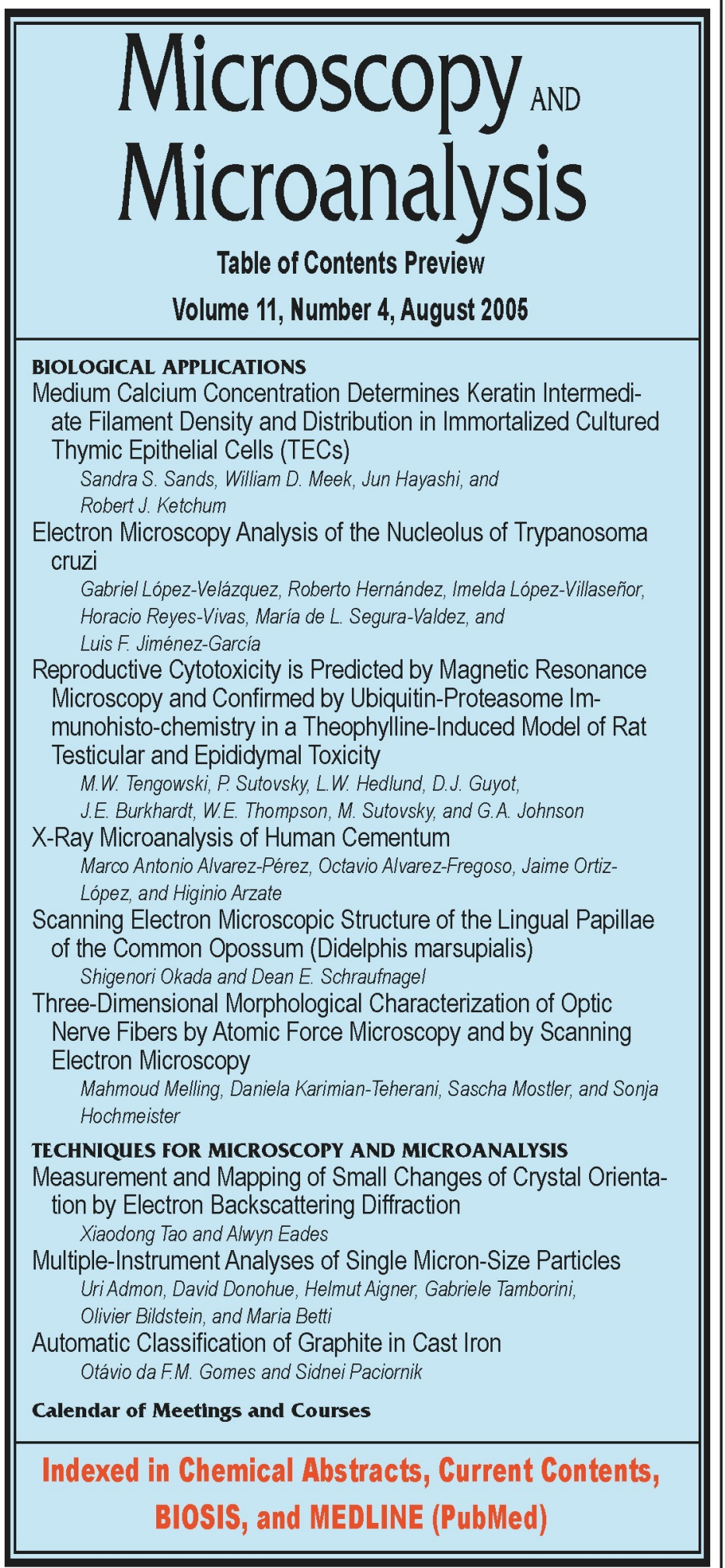

\section{The Problem: Black Square Deposits}

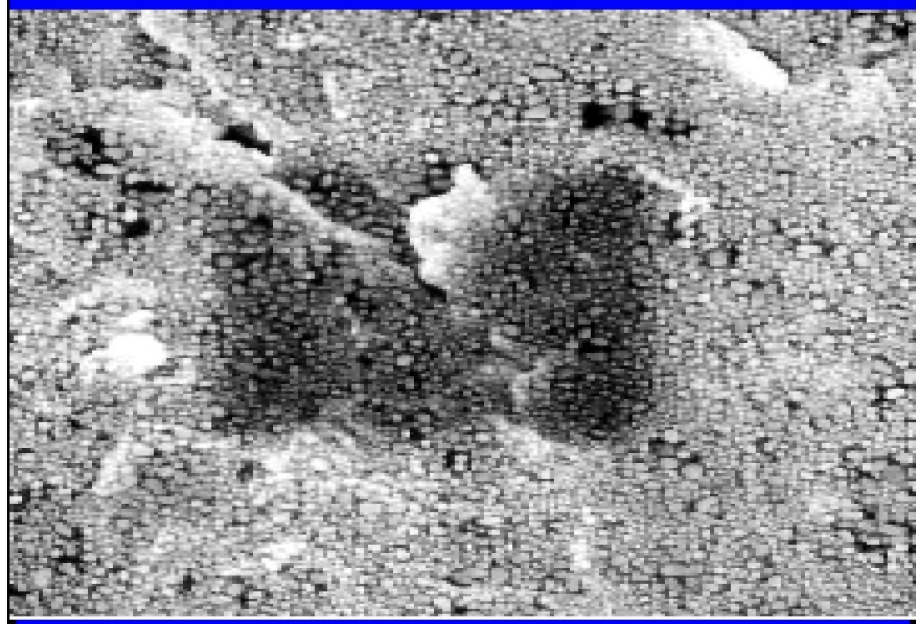

\section{The Remedy:}

$$
\begin{aligned}
& \text { Evactron }^{\circledR} \\
& \text { Gleaning }
\end{aligned}
$$
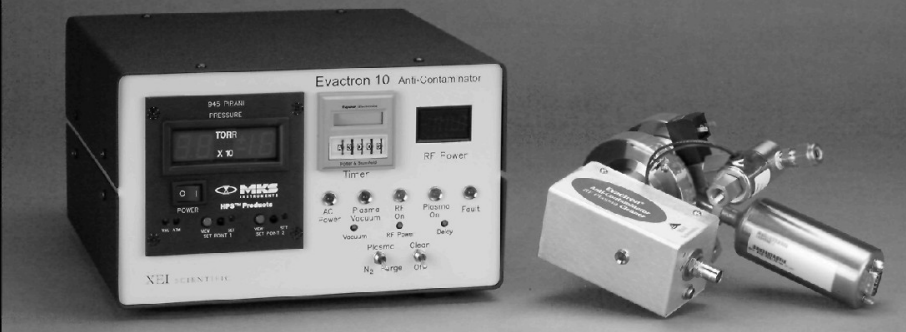

The Evactron ${ }^{\circledR}$ De-Contaminator with Oxygen Radical Source (ORS) mounts on your SEM or FIB.

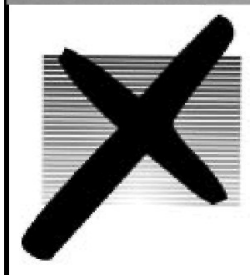

XEI sCientific, INC. 1735 East Bayshore Rd, Suite 29A Redwood City, CA 94063 (650) 369-0133, FAX (650) 363-1659 email:sales@Evactron.com www.EVACTRON.COM 